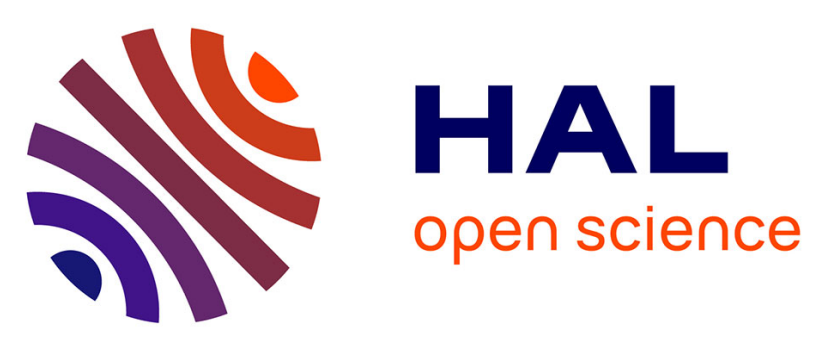

\title{
Bacterial Adherence to Plant and Animal Surfaces Via Adhesin-Lipid Interactions
}

\author{
Claire Rossi, Hélène Cazzola, Nicola Holden, Yannick Rossez
}

\section{To cite this version:}

Claire Rossi, Hélène Cazzola, Nicola Holden, Yannick Rossez. Bacterial Adherence to Plant and Animal Surfaces Via Adhesin-Lipid Interactions. Health Consequences of Microbial Interactions with Hydrocarbons, Oils, and Lipids, Springer International Publishing, pp.1-21, 2019, 10.1007/978-3-31972473-7_13-1. hal-02322704

\section{HAL Id: hal-02322704 https://hal.science/hal-02322704}

Submitted on 8 Jul 2021

HAL is a multi-disciplinary open access archive for the deposit and dissemination of scientific research documents, whether they are published or not. The documents may come from teaching and research institutions in France or abroad, or from public or private research centers.
L'archive ouverte pluridisciplinaire HAL, est destinée au dépôt et à la diffusion de documents scientifiques de niveau recherche, publiés ou non, émanant des établissements d'enseignement et de recherche français ou étrangers, des laboratoires publics ou privés. 


\title{
Bacterial Adherence to Plant and Animal Surfaces Via Adhesin-Lipid Interactions
}

\author{
Claire Rossi, Hélène Cazzola, Nicola J. Holden, and Yannick Rossez
}

\section{Contents}

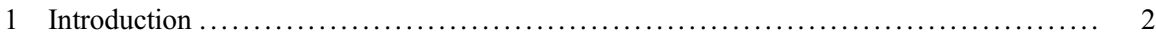

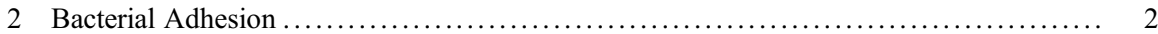

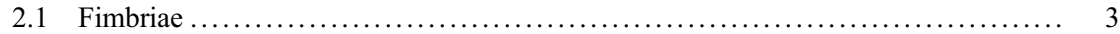

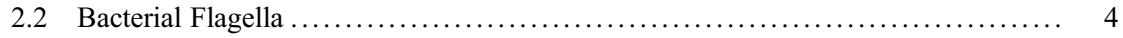

2.3 Other Adhesins and Bacterial Toxins ................................... 5

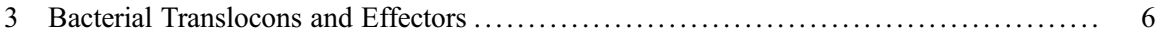

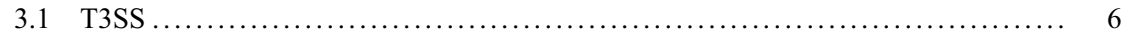

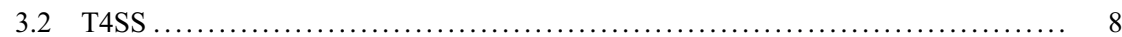

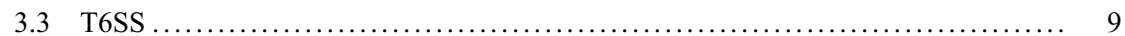

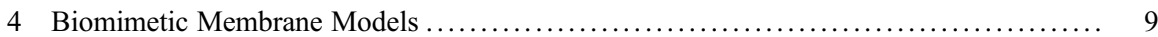

4.1 Liposomes and Bacterial Interaction .................................. 10

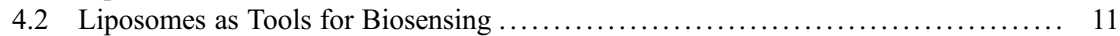

4.3 Bacterial Toxin Interaction with Tethered Bilayer Models ...................... 12

4.4 Whole-Bacteria Interaction with Membrane Models ......................... 14

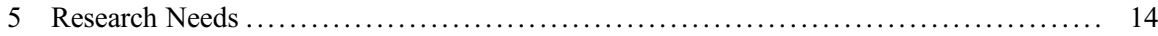

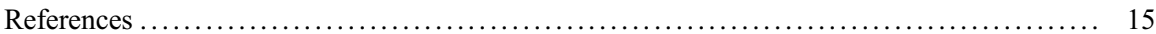

\section{Abstract}

The plasma membrane acts as one of the first lines of defense by establishing a physical barrier against microbes. Nevertheless, bacteria have developed a range of strategies to invade the host tissues efficiently. In this chapter, we focus on this understudied area and describe how bacteria target or redirect host membrane

C. Rossi · H. Cazzola $\cdot$ Y. Rossez ( $\square)$

UMR7025 CNRS Enzyme and Cell Engineering Laboratory, Sorbonne Universités, Université de Technologie de Compiègne, Compiègne, France

e-mail: Claire.Rossi@utc.fr; Helene.Cazzola@utc.fr; Yannick.Rossez@utc.fr

\section{N. J. Holden}

Cellular and Molecular Sciences, James Hutton Institute, Dundee, Scotland, UK e-mail: Nicola.Holden@hutton.ac.uk 
lipids. Domains enriched in sterols and sphingolipids, denominated membrane rafts, in particular, have been reported to be exploited by numerous bacterial pathogens. We will first describe the different strategies employed by bacteria to specifically target the PM via membrane rafts and non-raft counterpart domains; and secondly, we illustrate how development of biomimetic membranes has identified bacterial mechanisms of interaction.

\section{$1 \quad$ Introduction}

The cellular membrane is responsible for cell compartmentalization that coordinates the transport of molecules, cell signaling, or pathogen recognition. Most of these mechanisms are dependent on intricate interactions between proteins and lipids. The most abundant lipids are glycerolipids, sphingolipids, and sterols in animals and plants. Glycerolipids and sphingolipids are categorized into different lipid classes based on the chemical structures of their head groups and each class varies in its fatty acid content due to the degree of unsaturation, chain length, and linkage (Harayama and Riezman 2018). To add to this complexity, these molecules are not homogenously distributed across membranes, resulting in a spatial organization of the lipid landscape of the cell plasma membrane (PM). Sphingolipids have a specific physical location within plant and animal membranes. They are mainly located in the outer leaflet of the PM, whereas the glycerophospholipids like phosphatidylinositol (PI), phosphatidylserine (PS), and phosphatidylethanolamine (PE) occur mainly in the inner leaflet (van Meer and de Kroon 2011; Cacas et al. 2016). Pathogenic bacteria can overcome and even exploit this diversity and complex organization by directly binding interactions to different lipid classes from different cell-surface expressed appendages.

\section{$2 \quad$ Bacterial Adhesion}

Bacteria adhere to many surfaces for successful colonization of hosts and as a survival mechanism in the environment. Macromolecules exposed on the bacterial surface involved in adhesion are referred to as adhesins: a generic designation describing various extracellular structures, ranging from outer membrane proteins to a variety of monomeric or polymeric proteinaceous surface appendages (Kline et al. 2009). The best-studied cell appendages, exclusively found in Gram-negative bacteria, are the chaperone-ushered fimbriae (also known as pili). Adhesins typically recognize specific targets thus enabling the bacterium to bind on a specific surface, which results in tropism towards particular tissue types. Many different types of fimbriae have been described but the prototypical fimbriae are Type 1 (Ofek et al. 1977) in Escherichia coli and P fimbriae found in uropathogenic E. coli (UPEC) (Dodson et al. 2001) which mediate binding to specific receptors enriched in the bladder and upper urinary tract, respectively. Over the years, a large number of 


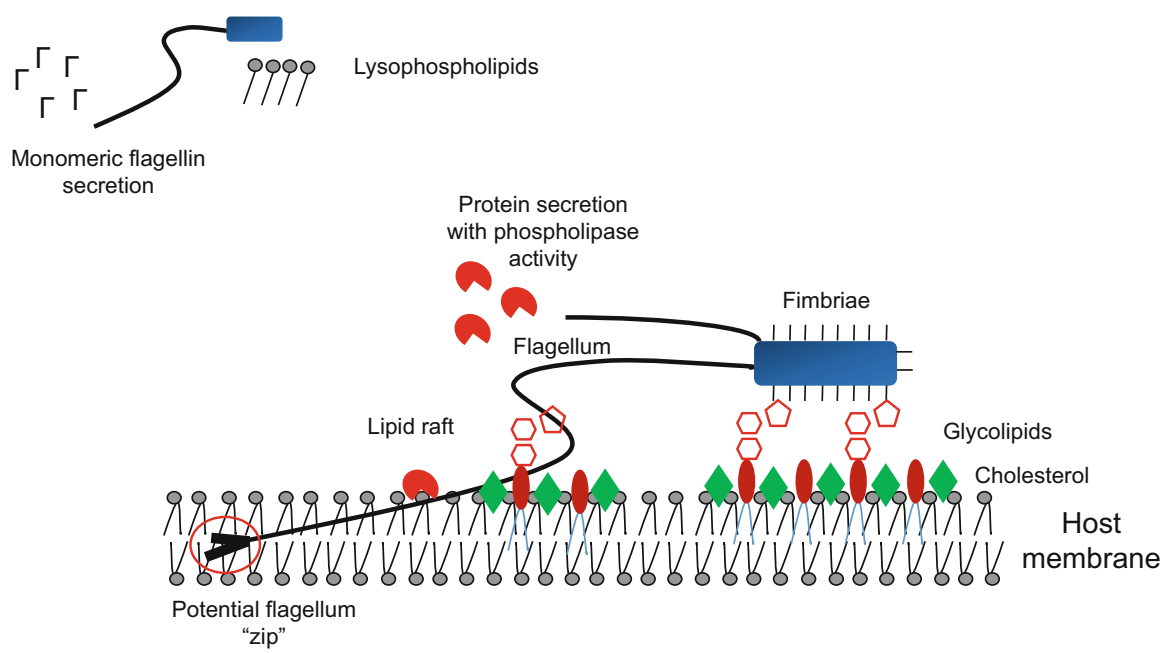

Fig. 1 Bacterial adhesion on host plasma membrane

adhesive proteins have been identified and, for some of them, their affinity for PM lipidic molecules (Fig. 1).

\subsection{Fimbriae}

Fimbriae are adhesive hair-like organelles expressed on the surface of bacteria, generally with an adhesin at the tip (Pizarro-Cerdá and Cossart 2006). They are exported to the bacterial surface by either a chaperone-usher set of proteins (Costa et al. 2015) or by a type IV or type V pili secretion (T4p or T5p) apparatus consisting each of several proteins (Hospenthal et al. 2017). Although bundle-forming pilus (BPF) of enteropathogenic E. coli (EPEC) has been shown to bind directly to PE (Khursigara et al. 2001), most fimbriae have a lectinic adhesin that binds a carbohydrate component of glycolipids. For example, the PapG adhesin of pyelonephritisassociated (P) pilus binds to $\alpha$-D-galactopyranosyl-( $1-4)-\beta$-D-galactopyranoside from a type of glycosphingolipid termed globoside, enriched in upper urinary tract cells (Väisänen et al. 1981; Dodson et al. 2001). Several other bacterial species like Streptococcus pneumoniae, Klebsiella pneumoniae, Pseudomonas aeruginosa, Haemophilus influenzae, and Staphylococcus aureus have been identified to bind specifically to this carbohydrate harbored by glycosphingolipids ganglioside, fucosylasialo-GM1, asialoGM1, and asialo-GM2 (Krivan et al. 1988). Interestingly, candidate adhesins involved in glycosphingolipid recognition have not yet been identified for these species, implicating potentially uncharacterized fimbriae gene clusters. Until recently, $P$. aeruginosa T4p was described to bind the GalNAc $\beta 1-4 \mathrm{Gal}$ moiety of the asialo-GM1 and asialo-GM2 via the adhesin PilA (Lee et al. 1994). However, the interaction was shown to be context dependent since 
binding to glycosphingolipids only occurred with sheared T4p organelles, and not when they are expressed from the $P$. aeruginosa bacterial surface (Emam et al. 2006). S-fimbriae found in E. coli adheres to galactosyl ceramide, lactosyl ceramide, and sulphated galactocerebroside (or sulphatide) via the SfaA adhesin protein (Prasadarao et al. 1993). Sulphatide can be found on different type of animal tissues and was first isolated from human brain meningeal tissue (Eckhardt 2008), hence these bacteria are often associated with meningitis (Parkkinen et al. 1988). The lipid plays a role in bacterial adhesion for a number of bacterial species like Actinobacillus pleuropneumoniae, Bordetella pertussis, and Campylobacter jejuni (Takahashi and Suzuki 2012). For transmembrane glycoproteins, their surrounding lipidic environment is indirectly involved for adhesion and invasion of target cells. FimH encoded by Type 1 fimbriae binds to mannosylated proteins in the urinary tract and needs lipid rafts for E. coli penetration of bladder epithelium (Duncan et al. 2004). In contrast, binding some FimH variants to plant tissue occurs via recognition of hemicellulosic $\beta$-(1-4) mannans or $N$-linked glycoproteins in plant cell walls (Marshall et al. 2016).

\subsection{Bacterial Flagella}

The flagellum is a major organelle present in the majority of bacterial species. This self-assembly protein machinery is mostly used for motility and chemotaxis (Evans et al. 2014). Flagellin, a globular protein forming the flagellum, is recognized by specialized host cell surface receptors such as Toll-like receptor 5 (TLR5) in animals or FLS2 in plants, and because of its virtual ubiquity in bacteria, it is a potent immunogen. Microbe perception occurs on flagellin binding to these receptors, triggering innate immunity activation (Rossez et al. 2015). However, the flagellum has also been identified as an adhesin. Flagella are capable of binding different surfaces including lipids, aided in reaching PM by their length (Rossez et al. 2015). Flagella from Salmonella enterica serovar Typhimurium have been shown to have an affinity for cholesterol from studies using cholesterol-coated surfaces. This surprising finding required verification that fimbrial adhesins, such as type 1 fimbriae, were not involved (Crawford et al. 2010). Different E. coli flagella serotypes have been demonstrated to bind negatively charged membrane lipids (phospholipids and sulpholipids) in planta (Rossez et al. 2014), where it is thought that the length and flagellar rotation help to penetrate the plant cell wall to the underlying PM. $P$. aeruginosa flagellum preferentially adheres on the gangliosides GM1 and aGM1. However, binding is thought to occur as an early step in adhesion before fimbriae adhesion (Feldman et al. 1998), which was supported by the finding that flagellar adhesion to ganglioside is a major factor for TLR activation (Adamo et al. 2004). Cellular stress-induced changes have been shown to influence pathogen interactions with epithelial cells, where stress (e.g., oxidative stress) activates acid sphingomyelinase, which induces PM remodeling, and prevents non-motile bacterial Shigella flexneri binding to the cells. In contrast, flagellated bacteria (S. enterica) can overcome this lipid remodeling process by accumulating at the remaining 
sphingolipid rich domains (Tawk et al. 2018). Hydrophobicity has also been shown to play a role in how bacterial flagella adhere, such that flagella can bind loosely to hydrophilic surfaces, whereas on hydrophobic surfaces, initial attachment is loose but increases progressively. This occurs apparently through conformational changes to the flagella, which may "zip" onto the surface and form stronger bonds with the hydrophobic surface (Friedlander et al. 2015). This work gives some insight on how bacterial flagella could interact with PM but needs to be tempered by the fact that PM are charged and composed by amphiphilic molecules.

Host lipids are not only used by bacteria to adhere on PM but can also serve to indicate the presence of the host cell to the pathogen via intermediate phospholipids like lysophospholipids. For example, in a form of host innate immunity regulation, secretion of $S$. enterica monomeric flagellin is induced by host-produced lysophospholipids to allow pathogen sensing, since only monomeric and polymerized flagellin trigger immune response via TLR-5 binding (Subramanian and Qadri 2006). For some species, secretion of non-flagellin proteins occurs via the flagella apparatus, similar to that of the non-flagellar type three-secretion system (T3SS). For example, in Yersinia enterocolitica, YplA, a non-flagellar protein has been shown to be secreted by the flagellar type III secretion system and is associated with a phospholipase activity (Schmiel et al. 1998; Young et al. 1999). In Campylobacter jejuni, which lacks non-flagellar T3SS, flagella-mediated secretion has been demonstrated for FlaC, an adhesin that facilitates binding to human cells (Song et al. 2004). This flagellin-like protein is secreted in order to specifically modulate host responses via TLR5 activation (Faber et al. 2016).

\subsection{Other Adhesins and Bacterial Toxins}

Besides appendages on bacterial surface, some outer membrane proteins can mediate adhesion to host surfaces. The outer membrane protein MAM7 (multivalent adhesion molecule) present in several Gram-negative bacteria has been shown to bind to fibronectin and phosphatidic acid (PA). Their adhesion can occur concurrently, although PA is fundamental to obtain a stable binding to the host and a basic residue (mce2 Lys-166) seems to be essential (Krachler et al. 2011). The rickettsial Outermembrane protein $\mathrm{B}(\mathrm{rOmpB})$ from the obligate intracellular tick-borne pathogen Rickettsia conorii facilitates both binding to and invasion of non-phagocytic mammalian cells, requiring complex regulation between several partners for internalization. rOmpB targets a $70 \mathrm{kDa}$ protein, $\mathrm{Ku} 70$, found at the host $\mathrm{PM}$ and in association with cholesterol-enriched lipid rafts. For internalization, three other proteins are needed: E3 ubiquitin ligase (c-Cbl), clathrin, and caveolin-2 (Martinez et al. 2005; Chan et al. 2009).

There are significant evidences showing bacterial toxins bind to membrane lipid to form pores. Some toxins target specifically membrane lipid raft to penetrate the cell. The best described is the cholera toxin B-subunit (CTxB), the membranebinding subunit of cholera toxin, which is used as a marker of lipid raft (Day and Kenworthy 2015). This toxin secreted by Vibrio cholera has two subunits: The 
CTxA with an enzymatic activity to $\mathrm{G}$ protein which activates adenylate cyclase and a homopentameric CTxB which binds to GM1 gangliosides (van Heyningen 1974). For complete reviews on bacterial toxins and pore formation see Geny and Popoff (2006); Tilley and Saibil (2006); Los et al. (2013).

\section{Bacterial Translocons and Effectors}

To successfully colonize host tissues, many bacterial pathogens have evolved multiple protein secretion systems. Bacteria use 12 distinct classes of protein secretion systems to export proteins through their multilayered cell envelope (Green and Mecsas 2016). Some of the secretion systems can translocate proteins, termed bacterial effectors, into host cells, which in some cases target PM lipids (Geissler 2012). For this, they need to target host PM to form a pore or to go through after structural contraction (Fig. 2). Of the systems described above that directly interact with host lipids, flagella are ancestrally related to the Type 3 secretion system (T3SS); and OMP are exported via Type 1 secretion systems (T1SS) (Costa et al. 2015). The T3SS, type IV secretion system (T4SS) and type VI secretion system (T6SS), found mainly in Gram-negative bacteria, interact directly with and/or cross the host PM (Costa et al. 2015).

\section{$3.1 \quad$ T3SS}

The type 3 secretion system (T3SS) is used by both plant and animal pathogens to deliver effector proteins into host cells, via a molecular syringe, to promote host interactions, pathogenic, beneficial, or commensal. The T3SS needle complex encoded by animal pathogens is shorter than that of plant pathogens, with a length of 20-40 nm whereas more than $100 \mathrm{~nm}$ is required to penetrate the thick plant cell wall (CW) (Ji and Dong 2015). The morphological and protein subunit similarities between the T3SS and the bacterial flagellar system components indicate that these systems are homologs, arising from a common evolutionary origin (Macnab 1999; Pallen and Gophna 2007). The flagellar apparatus is thought to be the evolutionary precursor of T3SS, which has diversified over a series of gene duplications, losses, and acquisitions and has evolved the ability to deliver effectors to eukaryotic cells (Nguyen et al. 2000; Saier 2004; Abby and Rocha 2012). To inject T3SS effectors, a pore-forming multi-protein organelle, termed a translocon is inserted into host PM upon contact with host cell. Three types of protein tanslocons allow delivery of effectors: two are hydrophobic and one hydrophilic. The hydrophobic translocators are subdivided in two categories, one with only one transmembrane (TM) domain and the other with two TM domains. Due to their TM domains, these proteins insert in the host PM to form the translocon. The hydrophilic protein connects the membrane-inserted translocon and the tip of the T3SS needle (Mattei et al. 2011; Deng et al. 2017). In animals cells, the pore formation process requires transmembrane protein CCR5 (chemokine [C-C motif] receptor 5) in order to modulate 


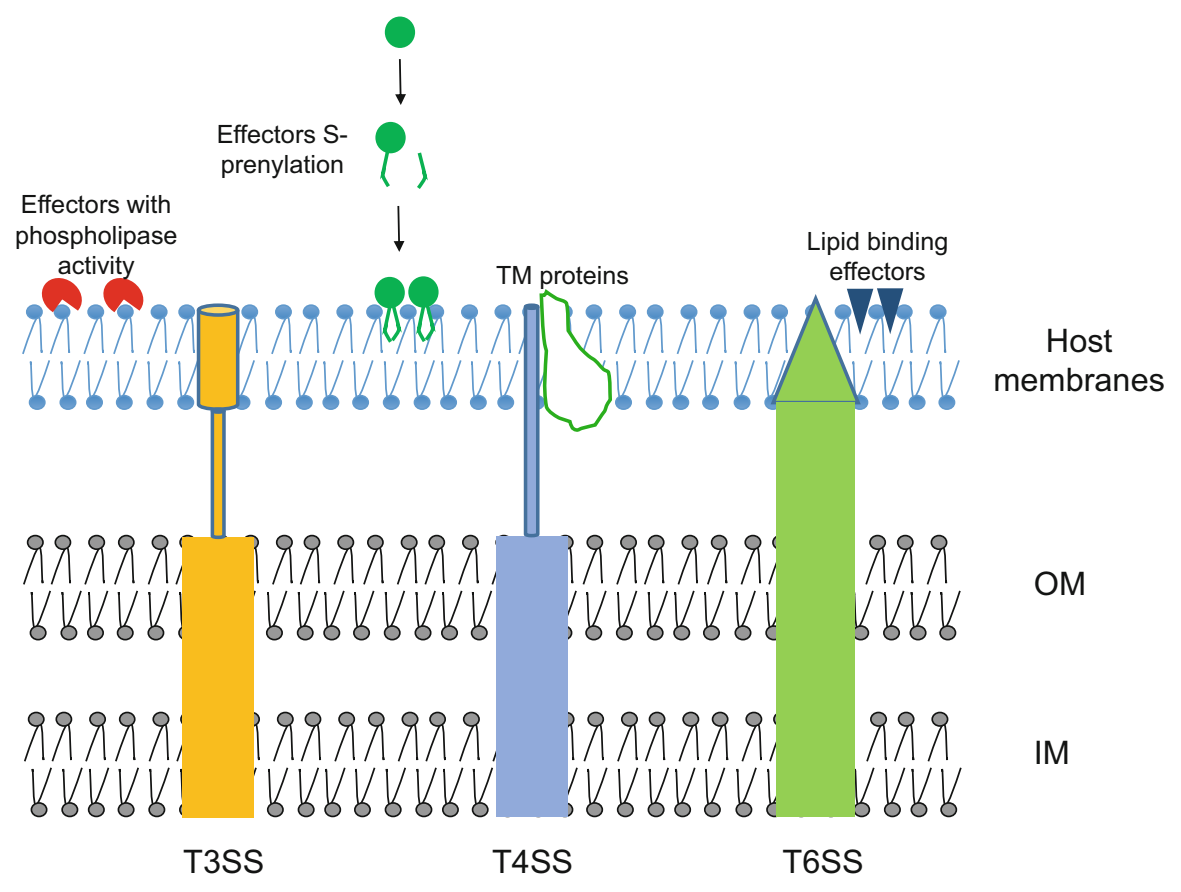

Fig. 2 Secretion systems, plasma membrane, and effectors

cytoskeletal activities and allow hydrophobic translocon assembly (Sheahan and Isberg 2015). In plants, T3SS accessories proteins are needed to degrade the $\mathrm{CW}$ and facilitate access for translocon to PM (Charkowski et al. 1998; Kim and Beer 1998). IpaB, in Shigella, is one TM domain protein located on the tip of T3SS and binds to the hyaluronan receptor CD44. This interaction is impacted by depletion of cholesterol or sphingolipids from the epithelial cell PM (Lafont 2002; Van Der Goot et al. 2004). Enteropathogenic E. coli (EPEC) secrete their own receptor via the T3SS, the translocated intimin receptor (Tir). PM embedded Tir then binds to the EPEC adhesin intimin and induces intimate attachment between the bacterial and host cells (Hartland et al. 1999).

T3SS effectors can target host PM directly by degrading phospholipids like PI, to facilitate host manipulation or colonization. PI lipids are implicated in a multitude of role from actin cytoskeleton arrangements to vesicle trafficking and are the target of pathogenic bacteria; see Hilbi (2006) for review. An effector from Shigella flexneri, the inositol phosphate phosphatase IpgD, hydrolyzes phosphatidylinositol-4,5bisphophate $\left(\mathrm{PIP}_{2}\right)$ to produce phosphatidylinositol 5-monophosphate (PI5P). This leads to actin reorganization promoting bacteria entry (Konradt et al. 2011). Others effectors from a number of bacteria (Ham et al. 2011) such as Mycobacterium tuberculosis (Beresford et al. 2007), S. enterica (Norris et al. 1998; Marcus et al. 2001), and V. parahaemolyticus (Broberg et al. 2010) are known to manipulate host membranes in comparable ways. 
Effectors from intracellular pathogens like S. enterica can modify phagosomes' membranes to prevent fusion with host lysosomes and then to replicate inside host cells. For example, SifA contains Cys-aliphatic-aliphatic-X (CAAX) motif in its last $\mathrm{C}$-terminal residues. When translocated, this motif is isoprenylated and S-acylated by eukaryotic cells. S-prenylation covalently adds isoprene groups, mainly farnesyl (15-carbon) and geranylgeranyl (20-carbon), to cysteine residues. These modifications increase the effector hydrophobicity and anchors them at the membrane (Boucrot et al. 2003; Reinicke et al. 2005).

\section{$3.2 \quad$ T4SS}

Like the T3SS, the type 4 secretion system (T4SS) can also reach host PM to transfer material proteins into the cytoplasm of eukaryotic cells and bacteria, although this includes DNA as well as proteins. The prototypical T4SS is from the phytopathogen, Agrobacterium tumefaciens (Grohmann et al. 2018). This bacterium uses the T4SS to transfer oncogenic T-DNA and different effectors to plant cells in order to induce crown gall disease (Salmond 1994). The T4SSs of Gram-negative bacteria appears to have evolved through consolidation of four functionally distinct structures. A hexameric ATPase, implicated in DNA/protein coupling and denominated type IV coupling protein (T4CP). Two membrane complexes needed for substrate passage across inner membrane or periplasm and outer membrane. They are named inner membrane complex (IMC) and outer membrane complex (OMC), respectively. The conjugative pilus is an example of the $\mathrm{OMC}$ and directly initiates contact with the host cell PM (Christie et al. 2014). Three proteins represent the pilus-assembly components: a transglycosylase (VirB1), a pilin (VirB2), and a pilus-tip (VirB5). To date, only VirB2 and VirB5 have been shown to be responsible for T4SS pilus adhesion on plant cells. Three related plant proteins with putative transmembrane domains, VirB2-interacting protein (BTI)-1, BTI2, and BTI3 were identified together with a membrane-associated GTPase, AtRAB8 to interact with VirB2 (Hwang and Gelvin 2004). Likewise, VirB5 has been described to target the transzeatin biosynthetic protein (Tzs) exposed to the plant cell surface (Aly et al. 2008). The human/animal pathogens, the best described T4SS is for Helicobacter pylori. The H. pylori VirB5 ortholog CagL, which was the first formally recognized bacterial carcinogen, binds specifically to a TM receptor, integrin $\alpha 5 \beta 1$ (Kwok et al. 2007). The H. pylori cytotoxin-associated gene A (CagA) interacts with PS on the host PM to promote CagA internalization (Murata-Kamiya et al. 2010). Specific host transmembrane proteins act as receptors for the $H$ pylori outer membrane protein HopQ. These proteins, called carcinoembryonic antigen-related cell adhesion molecules (CEACAMs), facilitate CagA translocation (Zhao et al. 2018). When translocated, CagA dysregulates the homeostatic signal transduction of gastric epithelial cells by shifting cell behavior. Similar to the T3SS effector SifA in S. enterica, the intravacuolar pathogen Legionella pneumophila T4SS effectors, AnkB when translocated in to the host are prenylated (Price et al. 2010). To replicate L. pneumophila needs to be intracellularly in an ER-associated compartment, the Legionella- 
containing vacuole (LCV). Approximately 300 effector proteins are translocated; some of the effectors anchor to membrane through PI4P or PI3P to promote the interaction of LCVs with host vesicles and organelles, to affect small GTPases or PImetabolizing enzymes see Steiner et al. (2018) for review.

\subsection{T6SS}

This versatile nanomachine was first described in 2006 in P. aeruginosa (Mougous et al. 2006). It delivers a wide arsenal of effector proteins directly into prokaryotic and eukaryotic target cells. T6SS share a bacteriophage-related tail structure anchored to the cell envelope by a membrane complex (Cherrak et al. 2000; Leiman et al. 2009). The T6SS tail tube is a structure made of different protein subunits that polymerize to form the contractile sheath. This tube wraps around the hemolysin co-regulated protein (Hcp) and dynamically propels a spike at its tip. This puncturing device is formed by Hcp and valine-glycine repeat protein $\mathrm{G}(\mathrm{VgrG})$. Then T6SS effectors are injected across bacterial membranes during this contraction mechanism (Basler et al. 2012). Effectors propelled by T6SS can affect host PM. In B. thailandensis, Vgrg-5 induce membrane fusion, either by the recruitment of other factors or by direct participation (Schwarz et al. 2014). In Vibrio cholerae, VasX is a secreted, lipid-binding protein, which can disrupt bacterial and host cell signaling. This T6SS protein can both kill and protect bacterial cells (Miyata et al. 2011, 2013). Antibacterial T6SS effectors target bacterial membrane with phospholipase activity in $P$. aeruginosa, Burkholderia thailandensis, or entero-aggregative $E$. coli (Russell et al. 2013; Flaugnatti et al. 2016).

\section{$4 \quad$ Biomimetic Membrane Models}

Here we describe application of biomimetic models as tools for the detection and characterization of bacterial proteins or whole bacteria interactions with host cell membranes. Membrane-based biomimetic systems have been developed to reconstitute and investigate biological events occurring at the cell membrane interface under controlled conditions. The complexity of the biological membranes makes direct investigation of their interaction with bacterial cells or compounds at the molecular level very challenging. In an attempt to overcome the limitations, lipid bilayer systems have been introduced as models of biological membranes. These models have been successfully used to study the interaction and the insertion of peptides, proteins, or whole organisms.

The simplest and most commonly used biomimetic models are vesicles and liposomes. These spherical lipid bilayers form an internal aqueous cavity and are classified according to their diameters into: Small Unilamellar Vesicles (SUV), which exhibit a mean diameter inferior to $100 \mathrm{~nm}$; Large Unilamellar Vesicles (LUV) have a diameter between 100 and $1000 \mathrm{~nm}$; whereas Giant Unilamellar Vesicles (GUV) have a size range of 1-200 $\mu \mathrm{m}$ of diameter. Due to their size, 


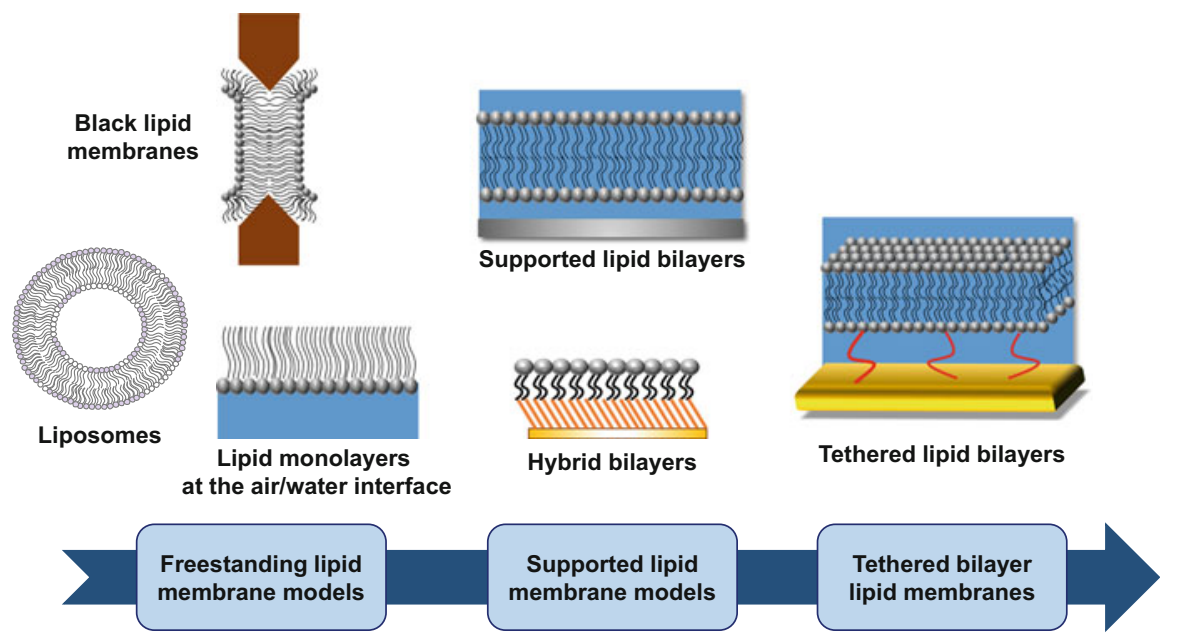

Fig. 3 Evolution of the lipid bilayer model architectures over time

GUVs are recognized as the best membrane model for mimicking the cell membrane curvature since animal and plant cells have a size ranging from 10 to $30 \mu \mathrm{m}$ and from 10 to $100 \mu \mathrm{m}$, respectively.

A variety of biomimetic membrane systems on solid support have been developed and coupled to surface sensitive techniques such as Surface Plasmon Resonance (SPR), Atomic Force Microscopy (AFM), quartz crystal microbalance, or neutronreflectometry. Among all the existing models, solid supported membranes provide higher stability than freestanding lipid models (Fig. 3). In 1984, Brian et al. pioneered a simplified method for the construction of supported planar membranes to study biological mechanisms (Brian and McConnell 1984). After this work, a variety of constructions on solid surface were developed including supported lipid bilayers (SLB) directly reconstituted on hydrophilic surfaces and tethered bilayers (tBLM) (Rebaud et al. 2014; Veneziano et al. 2017). Tethered bilayer lipid membranes were designed to overcome the limitations of SLB. They comprise a lipid bilayer spaced out from solid support by the use of molecular spacers or layers that intercalate between the substrate and the bilayer. This enables to study the incorporation or translocation of proteins without interference with the support (Tanaka and Sackmann 2005; Rossi and Chopineau 2007).

\subsection{Liposomes and Bacterial Interaction}

Liposomes are the simplest model system that mimic cell membranes. They offer the advantage of being very versatile as their lipid composition can be easily tailored to the question. They have been used to characterize the mode of interaction of bacteria with cell membranes and define the functional roles of the host cell membrane and bacterial components. LUVs were used in demonstration of the role of glycan- 
glycan interactions during the initial binding of Shigella dysenteriae to host cells. Outer membrane vesicles (OMV) containing lipopolysaccharide (LPS) were shown to be spontaneously, secreted by $S$. dysenteriae. In order to characterize the interaction of the O-antigen polysaccharide moiety of LPS, LUV with or without ganglioside GM1 were incubated with the OMVs and showed that the glycosylated part of GM1 from the host PM cells associated directly to the LPS. This phenomenon is involved in S. dysenteriae binding to host $\mathrm{CD}^{+} \mathrm{T}$ lymphocytes (Belotserkovsky et al. 2018).

Likewise, GUVs were used to implicate globotriaosylceramide Gb3-dependent invasion of Pseudomonas aeruginosa. The authors coined this mechanism "lipid zipper" where the galactophilic lectin (LecA) present in the bacterial outer membrane binds the carbohydrate moiety of $\mathrm{Gb} 3$ to mediate the entry of the bacteria. Microscopy analysis showed that the bacterial interaction with GUVs composed of $\mathrm{DOPC} / \mathrm{Chol} / \mathrm{Gb} 3$ was strictly driven by the lectin-Gb3 interaction and lead to the reorganization of membrane lipids into clusters with a negative membrane curvature that accompany the bacterial entry into GUVs (Eierhoff et al. 2014).

The importance of the nature of lipids involved in the bacteria/host cell membrane interaction can also be investigated with such biomimetic systems. Recently, it was shown by co-flotation assays that the signal peptide of the serine-rich repeat adhesin of Streptococcus gordonii interacts with anionic lipids of the host cell membrane. The electrostatic interaction was found to be a prerequisite for bacterial preprotein entry across cell membranes and the consecutive bacterial translocation (Spencer et al. 2018).

Liposomes were used in a separate example, to examine the effect of lipoarabinomannan (LAM), a component of the Mycobacterium tuberculosis cell wall, on membrane structure. This allowed the mechanistic basis of LAM to be identified for bacterial survival within macrophages. Using fluorescence resonance energy transfer (FRET), it was shown that, after insertion into the liposome bilayer, LAM inhibits the fusion of liposomes having a lipid composition mimicking the phagosomal membrane. Indeed a classic use of FRET is for monitoring lipid exchange or mixing and membrane fusion. Moreover, AFM imaging of lipid domains within a SLB exposed to LAM showed that LAM modifies the lipid distribution and packing, leading to a reorganization of lipid domains. This study neatly described how bacterial lipids can lead to the bacterial persistency within the immature phagosomal compartment of macrophages (Hayakawa et al. 2007).

\subsection{Liposomes as Tools for Biosensing}

The ability of some proteins such as toxins secreted by bacteria to insert in and then modify the physical properties of the lipid bilayer can be detected by biosensing systems. Zhou and co-authors developed a sensing methodology to detect pathogenic bacteria at an infected wound. The assay was based on the quantification of the release of a fluorescence dye from the lumen of liposomes upon exposure to bacterial lytic agents. Thus, the pathogenic state of the bacteria could be assessed (Zhou et al. 
2018). Based on the same principle, the membrane perturbing actions of several bacterial toxins were studied by monitoring the release of a fluorescent dye from liposomes. Recent applications are the vacuolating cytotoxin A (VacA), from $H$. pylori (Linn et al. 2018) or the Hemolysin A, the major virulence factor of Staphylococcus aureus. The authors have demonstrated that an inhibitor of ATP-gated purinergic receptors (P2XR) interferes with the function of a bacterial pore-forming protein through a direct inhibition of its binding to the membrane and oligomer formation (Schwiering et al. 2017).

\subsection{Bacterial Toxin Interaction with Tethered Bilayer Models}

Due to their covalent anchoring to the substrate, tethered bilayer lipid membranes (tBLMs) are considerably more robust than freestanding lipid bilayers. Among all the surface sensitive techniques that can be coupled to these models, electrochemical impedance spectroscopy (EIS) can be applied for studying the mode of interaction with lipid membranes of several membranotropic peptides (Atanasov et al. 2006). Indeed, depending on their molecular architecture, the tBLMs could provide a fluid lipid bilayer platform having a high resistivity, highly suited for quantifying the pore-forming ability of toxins produced by bacteria.

In EIS, an alternating voltage is applied over a range of frequencies and the resulting current is measured along with the time delay between a change in the applied potential and the resulting current. The resistance and phase difference between voltage and current changes can be fitted to an equivalent circuit composed of resistance and capacitance describing the electrical properties of the membrane (Fig. 4). For tBLMs, EIS measurements provide information about the quality of the lipid bilayer and charge transport processes across the membrane, for example, by extricated or incorporated channel proteins.

This approach was employed for the characterization of cyanotoxins. The action of a member of the microcystin (MC) family on the lipid membrane was investigated using tethered lipid bilayers (tBLMs). The cyanotoxin class of hepatotoxic MC is responsible for animal and human poisoning cases due to bioaccumulation in the liver. This study demonstrated that contrary to the original hypothesis, MC-Leucine Arginine (LR) membrane exposure leads only to small and transient variations in the membrane capacitance, showing that MC-LR is not able to accumulate in cell membranes and modify their integrity (Facey et al. 2019).

The combination of tBLMs and EIS measurements brings a more discriminative detection of the nature of the membrane damages caused by bacterial proteins than the liposome-based assays described above. An assay allowing the detection of toxins and bacterial enzymes was developed by coupling tBLMs with SPR spectroscopy and EIS (Tun et al. 2011). The tBLM used in this study is tethered by a mixture of 2,3-di-O-phytanyl-glycerol-1-tetraethylene glycol-D,L-lipoic ester lipid (DPhyTL) and cholesterol-pentaethyleneglycol. tBLMs integrating DPhyTL are well known to be dense lipid membranes with high electrical sealing properties (Naumann et al. 2003). The combination of these two techniques allows 


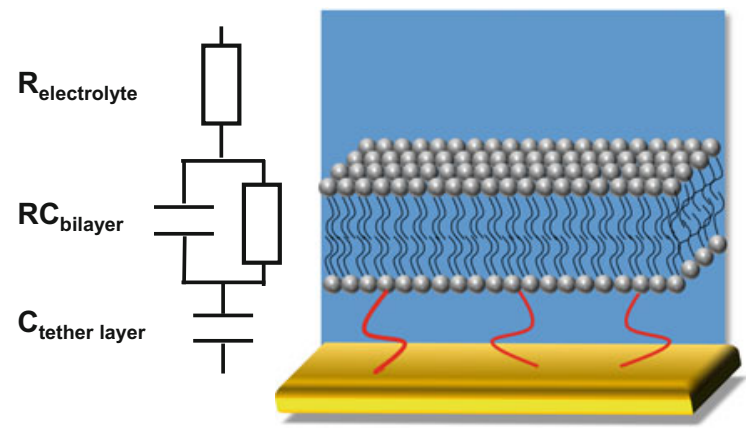

Fig. 4 Scheme of a tBLM (right) and an example of equivalent circuit modeling the resulting impedance data (left). The $\mathrm{R}(\mathrm{RC}) \mathrm{C}$-circuit consists of a RC element describing the bilayer in series with a capacitor $\left(\mathrm{C}_{\text {tether layer }}\right)$ corresponding to the tether layer and gold interface and an electrolyte resistance $\left(\mathrm{R}_{\text {electrolyte }}\right)$

investigators to differentiate between pore formation caused by pore-forming toxins or the lipid loss resulting from lipase action.

The design of tBLMs can be adapted in order to study more complex sequence of events involving bacterial toxins. The binding and the consecutive translocation of the adenylate cyclase toxin (CyaA) were successfully reconstituted and quantified under controlled conditions of calcium concentration and membrane potential (Veneziano et al. 2013). The CyaA toxin is one of the major virulence factors produced by Bordetella pertussis. It is a particularly complex protein of 1706residues and consisting of several active domains. Once translocated across the plasma membrane, the N-terminal catalytic domain (CD) of CyaA binds to calmodulin and catalyzes the production of supraphysiologic level of cAMP (O'Brien et al. 2017). A new tBLM construction was specifically developed to study the in vitro binding of CyaA to lipid membrane and the consecutive translocation of its CD across the lipid bilayer. The bilayer was assembled over a surface derivatized with the protein calmodulin in order to differentiate the external and internal aqueous compartments that are separated by the tethered bilayer. This particular design allows taking into account the underlying cytosolic compartment. It was demonstrated that the bilayer acts as a fluid and protein-impermeable barrier (Rossi et al. 2011). Surface plasmon resonance spectroscopy, application of a controlled membrane potential, and an enzymatic assay were combined in order to monitor the protein binding in real time and to quantify the enzymatic activity resulting from the $\mathrm{CD} / \mathrm{CaM}$ association. It was found that the translocation of the CyaA catalytic domain is strictly dependent on the presence of calcium and the application of a negative transmembrane potential across the tethered lipid bilayer (Veneziano et al. 2013). Beyond the study done on the CyaA toxin, this biomimetic design provides an unique tool to explore in vitro the molecular mechanisms of protein translocation across biological membranes in precisely defined conditions (Veneziano et al. 2013, 2017) 


\subsection{Whole-Bacteria Interaction with Membrane Models}

While the action on lipid membrane of proteins produced by bacteria have been studied using membrane models, very little attention has been applied for lipid bilayer systems by studying the interaction of whole bacteria with cell membrane structures.

To our knowledge, the membrane model structures employed for such studies have been exclusively vesicles. Functionalized GUVs $(\sim 15-60 \mu \mathrm{m})$ have been employed to monitor the interaction of whole bacteria with liposomal nanoparticles by electronic absorption spectroscopy and FRET. It was based on the interaction of the glucose molecules tagged on liposomes with the glycoproteins present on the surface of $E$. coli (Dogra et al. 2012). The same type of interaction was also exploited to investigate the propulsion of lipid vesicles using the strength of $E$. coli bacteria motility as a function of the size of the vesicles (Dogra et al. 2016). The glycolipidmediated interactions of bacteria with liposomes of different sizes (SUVs, LUVs, and GUVs) were evaluated by FRET microscopy. The authors demonstrated that SUVs and LUVs could be transported by bacteria while increasing their velocity, dependent on the size ratio between the bacteria and vesicle elements. In this study, any bacterial interaction with the liposomes composed of a pure lipid bilayer could be detected.

\section{$5 \quad$ Research Needs}

Bacterial adhesion to host tissue is a long-standing area of research, yet the interactions with lipids in this process remain an understudied topic. The major challenges that need to be addressed include:

- Understanding fatty acids (FA) function for bacterial adhesion and colonization. A small number of studies have been focused on the role of FA when bacteria invade host tissues although the physical state of the cells is dependent on the FA composition of the PM (Marsh 2010). Published studies are limited: in one study, use of membrane fluidizers like hexanol, benzyl alcohol, and 2-(2methoxyethoxy)ethyl 8-(cis-2-n-octylcyclopropyl)octanoate on human epithelial cell line. However, the approach doesn't fully address this question as it cannot discount side effects of the fluidizers on the cell lines (Ismaili et al. 1999). In another approach, cell lines incubated with different fatty acids and Lactobacillus spp illustrated the impact of FA on probiotic bacteria adhesion (Kankaanpää et al. 2001). Thus, by combining biomimetic membrane models and cell lines with such approaches, new mechanisms could be highlighted in host-pathogen interactions.

- Use transmembrane receptors with different plasma membrane lipid composition. The impact of different PM compositions on transmembrane proteins remains unknown for bacterial adhesion and immune response. The use of 
biomimetic membranes with controlled lipid composition in presence of reconstituted receptors could be an elegant approach to answer this question.

- Characterizing molecular recognition. To develop new molecular-based methods to combat bacterial infection, the scientific community needs to understand precisely which adhesins bind to specific PM lipids. Unfortunately, for most bacteria, this remains to be addressed.

- Cholesterol implication in bacterial colonization. Cholesterol-rich microdomains at the host PM have been identified in the internalization steps of several bacteria (Mañes et al. 2003). In most reports, cholesterol was removed with the appropriate drug. An alternative approach has employed mouse embryonic fibroblasts lacking the final enzyme required for cholesterol synthesis to investigate the role of cholesterol in bacterial penetration of host cells (Gilk et al. 2013). However, the precursor of cholesterol, desmosterol, that replaces cholesterol in the cellular PM of these mice may still participate in bacterial internalization (Rodríguez-Acebes et al. 2009). Therefore, additional work is needed to develop new strategies to study the real impact of cholesterol in bacterial adhesion and internalization.

Bacterial adhesion and manipulation of host membrane lipids still need to be better documented. Understanding how bacteria hijack PM organization will underlie new strategies to fight bacterial infection. While encouraging findings are emerging, major steps are still needed to better characterize the various strategies used by bacteria to target host lipids.

\section{References}

Abby SS, Rocha EPC (2012) The non-flagellar type III secretion system evolved from the bacterial flagellum and diversified into host-cell adapted systems. PLoS Genet:8. https://doi.org/10.1371/ journal.pgen. 1002983

Adamo R, Sokol S, Soong G et al (2004) Pseudomonas aeruginosa flagella activate airway epithelial cells through asialoGM1 and toll-like receptor 2 as well as toll-like receptor 5. Am J Respir Cell Mol Biol 30:627-634. https://doi.org/10.1165/rcmb.2003-0260OC

Aly KA, Krall L, Lottspeich F, Baron C (2008) The type IV secretion system component VirV5 binds to the trans-zeatin biosynthetic enzyme Tzs and enables its translocation to the cell surface of Agrobacterium tumefaciens. J Bacteriol 190:1595-1604. https://doi.org/10.1128/JB.01718-07

Atanasov V, Atanasova PP, Vockenroth IK et al (2006) A molecular toolkit for highly insulating tethered bilayer lipid membranes on various substrates. Bioconjug Chem 17:631-637. https:// doi.org/10.1021/bc050328n

Basler M, Pilhofer M, Henderson GP et al (2012) Type VI secretion requires a dynamic contractile phage tail-like structure. Nature 483:182-186. https://doi.org/10.1038/nature10846

Belotserkovsky I, Brunner K, Pinaud L et al (2018) Glycan-glycan interaction determines Shigella tropism toward human T lymphocytes. MBio 9:1-10. https://doi.org/10.1128/mBio.02309-17

Beresford N, Patel S, Armstrong J et al (2007) MptpB, a virulence factor from Mycobacterium tuberculosis, exhibits triple-specificity phosphatase activity. Biochem J 406:13-18. https:/doi. org/10.1042/BJ20070670

Boucrot E, Beuzón CR, Holden DW et al (2003) Salmonella typhimurium SifA effector protein requires its membrane-anchoring $\mathrm{C}$-terminal hexapeptide for its biological function. J Biol Chem 278:14196-14202. https://doi.org/10.1074/jbc.M207901200 
Brian AA, McConnell HM (1984) Allogeneic stimulation of cytotoxic T cells by supported planar membranes. Proc Natl Acad Sci U S A 81:6159-6163. https://doi.org/10.1073/pnas.81.19.6159

Broberg CA, Zhang L, Gonzalez H et al (2010) A Vibrio effector protein is an inositol phosphatase and disrupts host cell membrane integrity. Science 329:1660-1662. https://doi.org/10.1126/ science. 1192850

Cacas J-L, Buré C, Grosjean K et al (2016) Revisiting plant plasma membrane lipids in tobacco: a focus on sphingolipids. Plant Physiol 170:367-384. https://doi.org/10.1104/pp.15.00564

Chan YGY, Cardwell MM, Hermanas TM et al (2009) Rickettsial outer-membrane protein B $(\mathrm{rOmpB})$ mediates bacterial invasion through $\mathrm{Ku} 70$ in an actin, c-Cbl, clathrin and caveolin 2-dependent manner. Cell Microbiol 11:629-644. https://doi.org/10.1111/j.14625822.2008.01279.x

Charkowski AO, Alfano JR, Preston G et al (1998) The Pseudomonas syringae pv. tomato HrpW protein has domains similar to harpins and pectate lyases and can elicit the plant hypersensitive response and bind to pectate. J Bacteriol 180:5211-5217. https://doi.org/10.1063/1.2927482

Cherrak Y, Rapisarda C, Pellarin R et al (2000) Biogenesis and structure of a type VI secretion baseplate. https://doi.org/10.1038/s41564-018-0260-1

Christie PJ, Whitaker N, González-Rivera C (2014) Mechanism and structure of the bacterial type IV secretion systems. Biochim Biophys Acta, Mol Cell Res 1843:1578-1591. https://doi.org/ 10.1016/j.bbamcr.2013.12.019

Costa TRD, Felisberto-Rodrigues C, Meir A et al (2015) Secretion systems in Gram-negative bacteria: structural and mechanistic insights. Nat Rev Microbiol 13:343-359. https://doi.org/ 10.1038/nrmicro3456

Crawford RW, Reeve KE, Gunn JS (2010) Flagellated but not hyperfimbriated Salmonella enterica serovar typhimurium attaches to and forms biofilms on cholesterol-coated surfaces. J Bacteriol 192:2981-2990. https://doi.org/10.1128/JB.01620-09

Day CA, Kenworthy AK (2015) Functions of cholera toxin B-subunit as a raft cross-linker. Essays Biochem 57:135-145. https://doi.org/10.1042/bse0570135

Deng W, Marshall NC, Rowland JL et al (2017) Assembly, structure, function and regulation of type III secretion systems. Nat Rev Microbiol 15:323-337. https://doi.org/10.1038/ nrmicro.2017.20

Dodson KW, Pinkner JS, Rose T et al (2001) Structural basis of the interaction of the pyelonephritic E. coli adhesin to its human kidney receptor. Cell 105:733-743. https://doi.org/10.1016/S00928674(01)00388-9

Dogra N, Li X, Kohli P (2012) Investigating ligand-receptor interactions at bilayer surface using electronic absorption spectroscopy and fluorescence resonance energy transfer. Langmuir 28:12989-12998. https://doi.org/10.1021/la300724z

Dogra N, Izadi H, Vanderlick TK (2016) Micro-motors: a motile bacteria based system for liposome cargo transport. Sci Rep 6:1-9. https://doi.org/10.1038/srep29369

Duncan MJ, Li G, Shin J-S et al (2004) Bacterial penetration of bladder epithelium through lipid rafts. J Biol Chem 279:18944-18951. https://doi.org/10.1074/jbc.M400769200

Eckhardt M (2008) The role and metabolism of sulfatide in the nervous system. Mol Neurobiol 37:93-103. https://doi.org/10.1007/s12035-008-8022-3

Eierhoff T, Bastian B, Thuenauer R et al (2014) A lipid zipper triggers bacterial invasion. Proc Natl Acad Sci 111:12895-12900. https://doi.org/10.1073/pnas.1402637111

Emam A, Yu AR, Park HJ et al (2006) Laboratory and clinical Pseudomonas aeruginosa strains do not bind glycosphingolipids in vitro or during type IV pili-mediated initial host cell attachment. Microbiology 152:2789-2799. https://doi.org/10.1099/mic.0.28863-0

Evans LDB, Hughes C, Fraser GM (2014) Building a flagellum outside the bacterial cell. Trends Microbiol 22:566-572. https://doi.org/10.1016/j.tim.2014.05.009

Faber E, Gripp E, Maurischat S et al (2016) Novel immunomodulatory flagellin-like protein FlaC in Campylobacter jejuni and other Campylobacterales. mSphere 1:1-24. https://oi.org/10.1128/ mSphere.00028-15 
Facey JA, Steele JR, Violi JP et al (2019) An examination of microcystin-LR accumulation and toxicity using tethered bilayer lipid membranes (tBLMs). Toxicon 158:51-56. https://doi.org/ 10.1016/j.toxicon.2018.11.432

Feldman M, Bryan R, Rajan S et al (1998) Role of flagella in pathogenesis of Pseudomonas aeruginosa pulmonary infection. Infect Immun 66:43-51. https://iai.asm.org/

Flaugnatti N, Le TTH, Canaan S et al (2016) A phospholipase Alantibacterial type VI secretion effector interacts directly with the C-terminal domain of the VgrG spike protein for delivery. Mol Microbiol 99:1099-1118. https://doi.org/10.1111/mmi.13292

Friedlander RS, Vogel N, Aizenberg J (2015) Role of flagella in adhesion of Escherichia coli to abiotic surfaces. Langmuir 31:6137-6144. https://doi.org/10.1021/acs.langmuir.5b00815

Geissler B (2012) Bacterial toxin effector-membrane targeting: outside in, then back again. Front Cell Infect Microbiol 2:1-13. https://doi.org/10.3389/fcimb.2012.00075

Geny B, Popoff MR (2006) Bacterial protein toxins and lipids: pore formation or toxin entry into cells. Biol Cell 98:667-678. https://doi.org/10.1042/BC20050082

Gilk SD, Cockrell DC, Luterbach C et al (2013) Bacterial colonization of host cells in the absence of cholesterol. PLoS Pathog 9. https://doi.org/10.1371/journal.ppat.1003107

Green ER, Mecsas J (2016) Bacterial secretion systems: an overview. Microbiol Spectr 4:215-239. https://doi.org/10.1128/microbiolspec.VMBF-0012-2015.

Grohmann E, Christie PJ, Waksman G, Backert S (2018) Type IV secretion in Gram-negative and Gram-positive bacteria. Mol Microbiol 107:455-471. https://doi.org/10.1111/mmi.13896

Ham H, Sreelatha A, Orth K (2011) Manipulation of host membranes by bacterial effectors. Nat Rev Microbiol 9:635-646. https://doi.org/10.1038/nrmicro2602

Harayama T, Riezman H (2018) Understanding the diversity of membrane lipid composition. Nat Rev Mol Cell Biol 19:281-296. https://doi.org/10.1038/nrm.2017.138

Hartland EL, Batchelor M, Delahay RM et al (1999) Binding of intimin from enteropathogenic Escherichia coli to Tir and to host cells. Mol Microbiol 32:151-158. https://doi.org/10.1046/ j.1365-2958.1999.01338.x

Hayakawa E, Tokumasu F, Nardone GA et al (2007) A Mycobacterium tuberculosis-derived lipid inhibits membrane fusion by modulating lipid membrane domains. Biophys J 93:4018-4030. https://doi.org/10.1529/biophysj.107.104075

Hilbi H (2006) Modulation of phosphoinositide metabolism by pathogenic bacteria. Cell Microbiol 8:1697-1706. https://doi.org/10.1111/j.1462-5822.2006.00793.x

Hospenthal MK, Costa TRD, Waksman G (2017) A comprehensive guide to pilus biogenesis in Gram-negative bacteria. Nat Rev Microbiol 15:365-379. https://doi.org/10.1038/ nrmicro. 2017.40

Hwang HH, Gelvin SB (2004) Plant proteins that interact with VirB2, the Agrobacterium tumefaciens pilin protein, mediate plant transformation. Plant Cell 16:3148-3167. https://doi. org/10.1105/tpc.104.026476.1

Ismaili A, Meddings JB, Ratnam S, Sherman PM (1999) Modulation of host cell membrane fluidity: a novel mechanism for preventing bacterial adhesion. Am J Physiol 277:G201-G208. https:// doi.org/10.1152/ajpgi.1999.277.1.G201

Ji H, Dong H (2015) Key steps in type III secretion system (T3SS) towards translocon assembly with potential sensor at plant plasma membrane. Mol Plant Pathol 16:762-773. https://doi.org/ $10.1111 / \mathrm{mpp} .12223$

Kankaanpää PE, Salminen SJ, Isolauri E, Lee YK (2001) The influence of polyunsaturated fatty acids on probiotic growth and adhesion. FEMS Microbiol Lett 194:149-153. https://doi.org/ 10.1016/S0378-1097(00)00519-X

Khursigara C, Abul-Milh M, Lau B et al (2001) Enteropathogenic Escherichia coli virulence factor bundle-forming pilus has a binding specificity for phosphatidylethanolamine. Infect Immun 69:6573-6579. https://doi.org/10.1128/IAI.69.11.6573-6579.2001

Kim JF, Beer SV (1998) HrpW of Erwinia amylovora, a new harpin that contains a domain homologous to pectate lyases of a distinct class. J Bacteriol 180:5203-5210. https://jb.asm.org/ 
Kline KA, Fälker S, Dahlberg S et al (2009) Bacterial adhesins in host-microbe interactions. Cell Host Microbe 5:580-592. https://doi.org/10.1016/j.chom.2009.05.011

Konradt C, Frigimelica E, Nothelfer K et al (2011) The Shigella flexneri type three secretion system effector IpgD inhibits T cell migration by manipulating host phosphoinositide metabolism. Cell Host Microbe 9:263-272. https://doi.org/10.1016/j.chom.2011.03.010

Krachler AM, Ham H, Orth K (2011) Outer membrane adhesion factor multivalent adhesion molecule 7 initiates host cell binding during infection by Gram-negative pathogens. Proc Natl Acad Sci 108:11614-11619. https://doi.org/10.1073/pnas.1102360108

Krivan HC, Roberts DD, Ginsburg V (1988) Many pulmonary pathogenic bacteria bind specifically to the carbohydrate sequence GalNAc 1-4Gal found in some glycolipids. Proc Natl Acad Sci 85:6157-6161. https://doi.org/10.1073/pnas.85.16.6157

Kwok T, Zabler D, Urman S et al (2007) Helicobacter exploits integrin for type IV secretion and kinase activation. Nature 449:862-866. https://doi.org/10.1038/nature06187

Lafont F (2002) Initial steps of Shigella infection depend on the cholesterol/sphingolipid raftmediated CD44-IpaB interaction. EMBO J 21:4449-4457. https://doi.org/10.1093/emboj/ cdf457

Lee KK, Sheth HB, Wong WY et al (1994) The binding of Pseudomonas aeruginosa pili to glycosphingolipids is a tip-associated event involving the C-terminal region of the structural pilin subunit. Mol Microbiol 11:705-713. https://doi.org/10.1111/j.1365-2958.1994.tb00348.x

Leiman PG, Basler M, Ramagopal UA et al (2009) Type VI secretion apparatus and phage tailassociated protein complexes share a common evolutionary origin. Proc Natl Acad Sci 106:4154-4159. https://doi.org/10.1073/pnas.0813360106

Linn AK, Samainukul N, Sakdee S et al (2018) A Helicobacter pylori vacuolating cytotoxin A: mouse DHFR fusion protein triggers dye release from liposomes. Curr Microbiol 75:223-230. https://doi.org/10.1007/s00284-017-1369-9

Los FCO, Randis TM, Aroian RV, Ratner AJ (2013) Role of pore-forming toxins in bacterial infectious diseases. Microbiol Mol Biol Rev 77:173-207. https://doi.org/10.1128/ MMBR.00052-12

Macnab RM (1999) The bacterial flagellum: reversible rotary propellor and type III export apparatus. J Bacteriol 181:7149-7153. https://jb.asm.org/

Mañes S, Del Real G, Martínez-A C (2003) Pathogens: raft hijackers. Nat Rev Immunol 3:557-568. https://doi.org/10.1038/nri1129

Marcus SL, Wenk MR, Steele-Mortimer O, Finlay BB (2001) A synaptojanin-homologous region of Salmonella typhimurium $\mathrm{SigD}$ is essential for inositol phosphatase activity and Akt activation. FEBS Lett 494:201-207. https://doi.org/10.1016/S0014-5793(01)02356-0

Marsh D (2010) Structural and thermodynamic determinants of chain-melting transition temperatures for phospholipid and glycolipids membranes. Biochim Biophys Acta Biomembr 1798:40-51. https://doi.org/10.1016/j.bbamem.2009.10.010

Marshall J, Rossez Y, Mainda G et al (2016) Alternate thermoregulation and functional binding of Escherichia coli type 1 fimbriae in environmental and animal isolates. FEMS Microbiol Lett 363:1-8. https://doi.org/10.1093/femsle/fnw251

Martinez JJ, Seveau S, Veiga E et al (2005) Ku70, a component of DNA-dependent protein kinase, is a mammalian receptor for Rickettsia conorii. Cell 123:1013-1023. https://doi.org/10.1016/j. cell.2005.08.046

Matteï PJ, Faudry E, Job V et al (2011) Membrane targeting and pore formation by the type III secretion system translocon. FEBS J 278:414-426. https://doi.org/10.1111/j.17424658.2010.07974.x

Miyata ST, Kitaoka M, Brooks TM et al (2011) Vibrio cholerae requires the type VI secretion system virulence factor vasx to kill Dictyostelium discoideum. Infect Immun 79:2941-2949. https://doi.org/10.1128/IAI.01266-10

Miyata ST, Unterweger D, Rudko SP, Pukatzki S (2013) Dual expression profile of type VI secretion system immunity genes protects pandemic Vibrio cholerae. PLoS Pathog 9:1-18. https://doi.org/10.1371/journal.ppat.1003752 
Mougous JD, Cuff ME, Raunser S et al (2006) A virulence locus of Pseudomonas aeruginosa encodes a protein secretion apparatus. Science 312:1526-1530. https://doi.org/10.1126/ science. 1128393

Murata-Kamiya N, Kikuchi K, Hayashi T et al (2010) Helicobacter pylori exploits host membrane phosphatidylserine for delivery, localization, and pathophysiological action of the CagA oncoprotein. Cell Host Microbe 7:399-411. https://doi.org/10.1016/j.chom.2010.04.005

Naumann R, Schiller SM, Giess F et al (2003) Tethered lipid bilayers on ultraflat gold surfaces. Langmuir 19:5435-5443. https://doi.org/10.1021/la0342060

Nguyen L, Paulsen IT, Tchieu J et al (2000) Phylogenetic analyses of the constituents of type III protein secretion systems. J Mol Microbiol Biotechnol 2:125-144. https://www.karger.com/ Journal/Home/228391

Norris FA, Wilson MP, Wallis TS et al (1998) SopB, a protein required for virulence of Salmonella dublin, is an inositol phosphate phosphatase. Proc Natl Acad Sci 95:14057-14059. https://doi. org/10.1073/pnas.95.24.14057

O'Brien DP, Durand D, Voegele A et al (2017) Calmodulin fishing with a structurally disordered bait triggers CyaA catalysis. PLoS Biol 15:e2004486. https://doi.org/10.1371/journal. pbio. 2004486

Ofek I, Mirelman D, Sharon N (1977) Adherence of Escherichia coli to human mucosal cells mediated by mannose receptors. Nature 265:623-625. https://doi.org/10.1038/265623a0

Pallen M, Gophna U (2007) Bacterial flagella and type III secretion: case studies in the evolution of complexity. Genome Dyn 3:30-47. https://doi.org/10.1159/000107602

Parkkinen J, Korhonen TK, Pere A et al (1988) Binding sites in the rat brain for Escherichia coli S fimbriae associated with neonatal meningitis. J Clin Invest 81:860-865. https://doi.org/10.1172/ JCI113395

Pizarro-Cerdá J, Cossart P (2006) Bacterial adhesion and entry into host cells. Cell 124:715-727. https://doi.org/10.1016/j.cell.2006.02.012

Prasadarao NV, Wass CA, Hacker J et al (1993) Adhesion of S-fimbriated Escherichia coli to brain glycolipids mediated by $\mathrm{sfaA}$ gene-encoded protein of S-fimbriae. J Biol Chem 268:10356-10363. http://www.jbc.org/

Price CTD, Jones SC, Amundson KE, Abu Kwaik Y (2010) Host-mediated post-translational prenylation of novel Dot/Icm-translocated effectors of Legionella pneumophila. Front Microbiol 1:1-9. https://doi.org/10.3389/fmicb.2010.00131

Rebaud S, Maniti O, Girard-Egrot AP (2014) Tethered bilayer lipid membranes (tBLMs): interest and applications for biological membrane investigations. Biochimie 107:135-142. https://doi. org/10.1016/j.biochi.2014.06.021

Reinicke AT, Hutchinson JL, Magee AI et al (2005) A Salmonella typhimurium effector Protein SifA is modified by host cell prenylation and S-acylation machinery. J Biol Chem 280:14620-14627. https://doi.org/10.1074/jbc.M500076200

Rodríguez-Acebes S, de la Cueva P, Fernández-Hernando C et al (2009) Desmosterol can replace cholesterol in sustaining cell proliferation and regulating the SREBP pathway in a sterol- $\Delta^{24}$. reductase-deficient cell line. Biochem J 420:305-318. https://doi.org/10.1042/BJ20081909

Rossez Y, Holmes A, Wolfson EB et al (2014) Flagella interact with ionic plant lipids to mediate adherence of pathogenic Escherichia coli to fresh produce plants. Environ Microbiol 16:2181-2195. https://doi.org/10.1111/1462-2920.12315

Rossez Y, Wolfson EB, Holmes A et al (2015) Bacterial flagella: twist and stick, or dodge across the kingdoms. PLoS Pathog 11:e1004483. https://doi.org/10.1371/journal.ppat.1004483

Rossi C, Chopineau J (2007) Biomimetic tethered lipid membranes designed for membrane-protein interaction studies. Eur Biophys J 36:955-965. https://doi.org/10.1007/s00249-007-0202-y

Rossi C, Doumiati S, Lazzarelli C, Davi M, Meddar F, Ladant D, Chopineau J, Schnur JM (2011) A tethered bilayer assembled on top of immobilized calmodulin to mimic cellular compartmentalization. PLoS ONE 6 (4):e19101. https://doi.org/10.1371/journal.pone.0019101 
Russell AB, LeRoux M, Hathazi K et al (2013) Diverse type VI secretion phospholipases are functionally plastic antibacterial effectors. Nature 496:508-512. https://doi.org/10.1038/ nature 12074

Saier MH (2004) Evolution of bacterial type III protein secretion systems. Trends Microbiol 12:113-115. https://doi.org/10.1016/j.tim.2004.01.003

Salmond PCG (1994) Secretion of extracellular virulence factors by plant pathogenic bacterial. Annu Rev Phytopathol 32:181-200. https://doi.org/10.1146/annurev.py.32.090194.001145

Schmiel DH, Wagar E, Karamanou L et al (1998) Phospholipase A of Yersinia enterocolitica contributes to pathogenesis in a mouse model. Infect Immun 66:3941-3951. https://iai.asm.org/

Schwarz S, Singh P, Robertson JD et al (2014) VgrG-5 is a Burkholderia type VI secretion systemexported protein required for multinucleated giant cell formation and virulence. Infect Immun 82:1445-1452. https://doi.org/10.1128/IAI.01368-13

Schwiering M, Husmann M, Hellmann N (2017) P2X-receptor antagonists inhibit the interaction of S. aureus hemolysin a with membranes. Toxins (Basel) 9. https://doi.org/10.3390/ toxins 9100332

Sheahan KL, Isberg RR (2015) Identification of mammalian proteins that collaborate with type III secretion system function: involvement of a chemokine receptor in supporting translocon activity. MBio 6:1-13. https://doi.org/10.1128/mBio.02023-14

Song YC, Jin S, Louie H et al (2004) FlaC, a protein of Campylobacter jejuni TGH9011 (ATCC43431) secreted through the flagellar apparatus, binds epithelial cells and influences cell invasion. Mol Microbiol 53:541-553. https://doi.org/10.1111/j.1365-2958.2004.04175.x

Spencer C, Bensing BA, Mishra NN, Sullam PM (2018) Membrane trafficking of the bacterial adhesin GspB and the accessory Sec transport machinery. J Biol Chem 5. https://doi.org/ 10.1074/jbc.RA118.005657

Steiner B, Weber S, Hilbi H (2018) Formation of the Legionella-containing vacuole: phosphoinositide conversion, GTPase modulation and ER dynamics. Int J Med Microbiol 308:49-57. https://doi.org/10.1016/j.ijmm.2017.08.004

Subramanian N, Qadri A (2006) Lysophospholipid sensing triggers secretion of flagellin from pathogenic salmonella. Nat Immunol 7:583-589. https://doi.org/10.1038/ni1336

Takahashi T, Suzuki T (2012) Role of sulfatide in normal and pathological cells and tissues. J Lipid Res 53:1437-1450. https://doi.org/10.1194/jlr.R026682

Tanaka M, Sackmann E (2005) Polymer-supported membranes as models of the cell surface. Nature 437:656-663. https://doi.org/10.1038/nature04164

Tawk C, Nigro G, Rodrigues Lopes I et al (2018) Stress-induced host membrane remodeling protects from infection by non-motile bacterial pathogens. EMBO J 37:e98529. https://doi. org/10.15252/embj.201798529

Tilley SJ, Saibil HR (2006) The mechanism of pore formation by bacterial toxins. Curr Opin Struct Biol 16:230-236. https://doi.org/10.1016/j.sbi.2006.03.008

Tun TN, Cameron PJ, Jenkins ATA (2011) Sensing of pathogenic bacteria based on their interaction with supported bilayer membranes studied by impedance spectroscopy and surface plasmon resonance. Biosens Bioelectron 28:227-231. https://doi.org/10.1016/j.bios.2011.07.023

Väisänen V, Tallgren L, Helena Mäkelä P et al (1981) Mannose-resistant haemagglutination and P antigen recognition are characteristic of Escherichia coli causing primary pyelonephritis. Lancet 318:1366-1369. https://doi.org/10.1016/S0140-6736(81)92796-3

Van Der Goot FG, Van Nhieu GT, Allaoui A et al (2004) Rafts can trigger contact-mediated secretion of bacterial effectors via a lipid-based mechanism. J Biol Chem 279:47792-47798. https://doi.org/10.1074/jbc.M406824200

van Heyningen S (1974) Cholera toxin: interaction of subunits with ganglioside GM1. Science 183:656-657. https://doi.org/10.1126/science.183.4125.656

van Meer G, de Kroon AIPM (2011) Lipid map of the mammalian cell. J Cell Sci 124:5-8. https:// doi.org/10.1242/jcs.071233 
Veneziano R, Rossi C, Chenal A et al (2013) Bordetella pertussis adenylate cyclase toxin translocation across a tethered lipid bilayer. Proc Natl Acad Sci 110:20473-20478. https://doi.org/ 10.1073/pnas.1312975110

Veneziano R, Rossi C, Chenal A et al (2017) Synthesis and characterization of tethered lipid assemblies for membrane protein reconstitution (review). Biointerphases 12:04E301. https:// doi.org/10.1116/1.4994299

Young GM, Schmiel DH, Miller VL (1999) A new pathway for the secretion of virulence factors by bacteria: the flagellar export apparatus functions as a protein-secretion system. Proc Natl Acad Sci U S A 96:6456-6461. https://doi.org/10.1073/pnas.96.11.6456

Zhao Q, Busch B, Jiménez-Soto LF et al (2018) Integrin but not CEACAM receptors are dispensable for Helicobacter pylori CagA translocation. PLoS Pathog 14. https://doi.org/10.1371/ journal.ppat.1007359

Zhou J, Yao D, Qian Z et al (2018) Bacteria-responsive intelligent wound dressing: simultaneous in situ detection and inhibition of bacterial infection for accelerated wound healing. Biomaterials 161:11-23. https://doi.org/10.1016/j.biomaterials.2018.01.024 